\title{
Chemoradiation for Laryngeal Cancer: Present Scenario
}

\author{
${ }^{1}$ Adwaita Gore, ${ }^{2}$ Pushkal Dwiwedi, ${ }^{3}$ Boman Dhabhar, ${ }^{4}$ Tapan K Saikia \\ ${ }^{1}$ Senior Registrar, Department of Medical Oncology, Prince Aly Khan Hospital, Mumbai, Maharashtra, India \\ ${ }^{2}$ Clinical Fellow, Department of Medical Oncology, Prince Aly Khan Hospital, Mumbai, Maharashtra, India \\ ${ }^{3}$ Consultant Medical Oncologist, Department of Medical Oncology, Prince Aly Khan Hospital, Mumbai, Maharashtra, India \\ ${ }^{4}$ Consultant Medical Oncologist and Head of Medical Oncology, Department of Medical Oncology, Prince Aly Khan Hospital \\ Mumbai, Maharashtra, India
}

Correspondence: Adwaita A Gore, Senior Registrar, Department of Medical Oncology, Prince Aly Khan Hospital, Aga Hall Nesbit Road, Mazagaon, Mumbai-400010, Maharashtra, India, Phone: 9821262618/7738565220, e-mail: adygore@gmail.com

\begin{abstract}
The management of locally advanced squamous cell carcinoma of the larynx is complex. Data from recent clinical trials have refined the use of existing therapies, such as radiation therapy and chemotherapy, and providing new treatment options, such as cetuximab. Selecting the most appropriate treatment for an individual patient requires a multidisciplinary approach and careful assessment of the relative advantages and disadvantages of each treatment approach. Chemoradiation is more effective than conventional radiation therapy, but also more toxic. Cetuximab plus radiation therapy is more effective than radiation alone and does not substantially increase radiation-related toxicity. Multimodality approach for laryngeal cancer has shown better organ preservation rates and survival. Ideally, multidisciplinary teams should weigh all these factors when making individual treatment decisions. Data from current trials will help further optimize multimodality treatment for Laryngeal Cancer.
\end{abstract}

Keywords: Cetuximab, Radiotherapy, Chemoradiation, EGFR, Laryngeal cancer, Head and neck cancer.

\section{INTRODUCTION}

Radiation therapy in head and neck cancers was used earlier as local form of therapy when surgery was not feasible. Radiation therapy (RT) is used to control locally confined tumors with organ preservation. The ability of radiation alone to control soft tissue tumors is often limited by the tumor volume or the surrounding normal tissue tolerance to radiation. Cytotoxic chemotherapy drugs and biologic agents have been given before, during, or after RT in order to improve tumor responses. Several different approaches have been tested in an effort to integrate chemotherapy into definitive non-operative management of squamous cell head and neck cancer referred to as chemoradiation when administered together. In phase III randomized trials and in meta-analyses, the concomitant use of chemotherapy and radiation has proven to be the most consistently successful, and to produce both locoregional control and survival benefits when compared to radiation therapy alone.

Neoadjuvant or induction chemotherapy can be employed to decrease micrometastases and decrease tumor size prior to RT with the hopes of improving tumour control with RT and/or decreasing the amount of normal tissue irradiated.

Adjuvant chemotherapy is given after RT primarily to decrease systemic micro metastases. Concurrent chemoradiotherapy (chemo-RT) could enhance the sensitivity of tumor cells to RT while having no overlapping toxicities. This spatial cooperation was the initial rationale for combining chemotherapy and RT in which each agent had an independent mechanism of action at a different anatomic target. Since that time, concurrent chemoradiation has been used to enhance radio response locally within the primary tumor. Here, we review the recent clinical data in support of newer generation cytotoxic chemotherapies and systemic targeted agents in combination with RT.

\section{Molecular Basis of Combined Modality Therapy}

Combined modality therapy enhances RT or systemic therapies alone at both the tissue and cellular levels. At the tissue level, radiation can increase vascular permeability and allow greater drug delivery to the tumor as well as increase drug concentration by promoting drug retention within the 


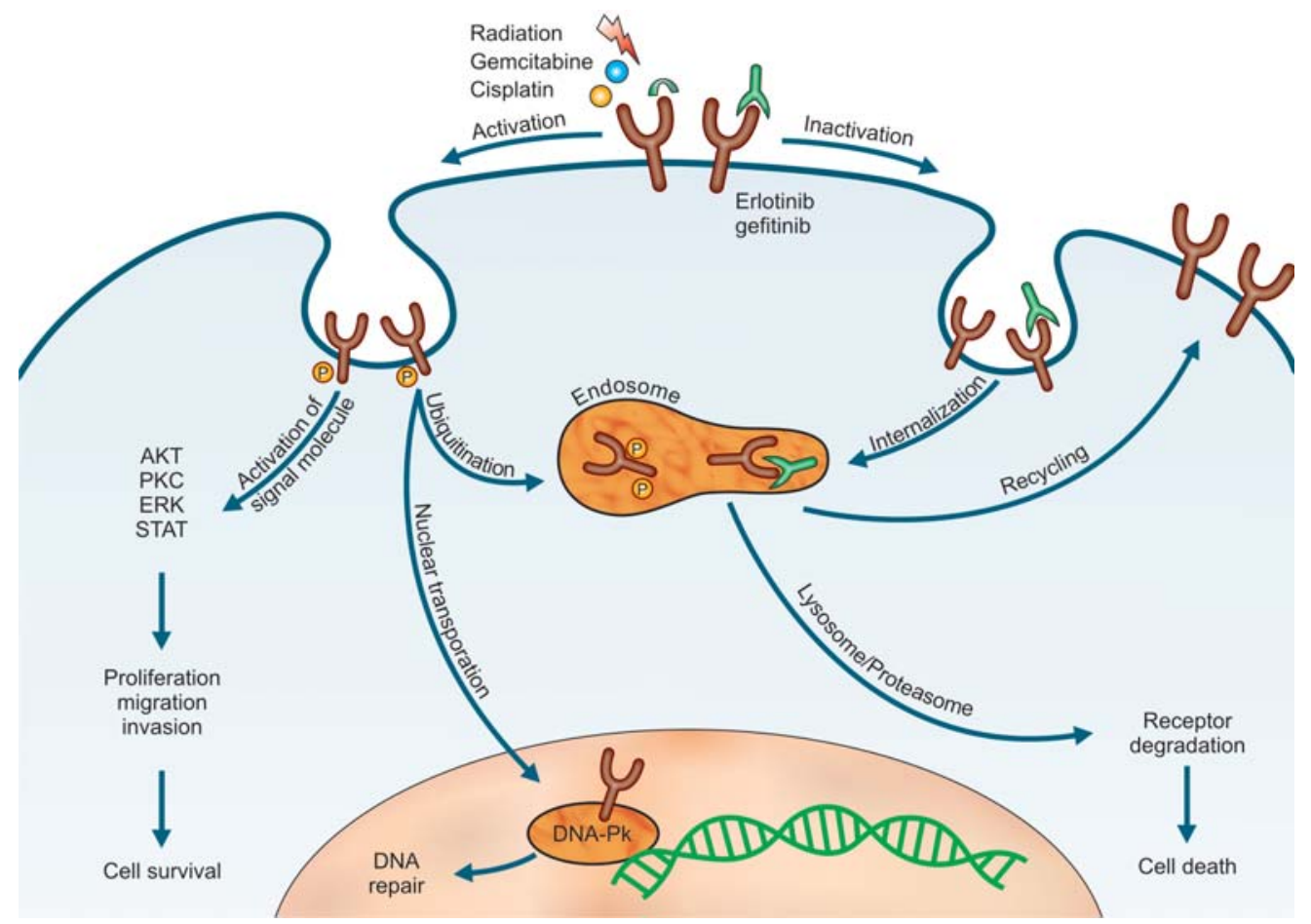

Fig. 1: The effects of radiation, chemotherapy, and targeted agents on EGFR signaling. After stimulation by irradiation or certain chemotherapeutic agents, epidermal growth factor receptor (EGFR) can activate downstream signaling pathways that can promote cell survival or cell death. In addition to stimulating the pathways activated by epidermal growth factor (EGF), radiation can trigger the translocation of phosphorylated EGFR (pEGFR) into the nucleus. This process coincides with the transport of Ku70/80 and protein phosphatase 1 into the nucleus (not shown), which results in increases in DNA-dependent protein kinase (DNAPK) levels, the repair of DNA-strand breaks, and cell survival. Cetuximab blocks nuclear transport of pEGFR; it binds to EGFR and causes endosome internalization, ultimately causing receptor degradation and cell death. Gefitinib and erlotinib bind to the intracellular ATP binding site of EGFR, thereby inhibiting unregulated EGFR signaling. Gemcitabine causes the phosphorylation of EGFR. In this case, EGFR phosphorylation initially activates Akt promoting cell survival, but subsequently promotes the ubiquitination $(\mathrm{Ub})$ of the receptor, which leads to its degradation along a proteosome or lysosome pathway. pEGFR degradation results in the down regulation of the survival signal pAkt, leading to apoptosis. Blocking EGFR degradation at various steps of this pathway reduces Gemcitabinemediated cytotoxicity. Whether an EGFR-activating insult leads to cell survival or cell death might ultimately be determined by the severity and duration of the stress. ${ }^{1}$

tumor. At the cellular level, systemic therapies can enhance radiation sensitivity by inhibiting DNA repair mechanisms enhancing oxygen radical formation to promote DNA double strand breaks, inhibiting progression through the cell cycle to lock cells in a radiosensitive phase, inducing apoptosis, and inhibiting cellular signaling cascades (Fig. 1).

Recent advances in molecular biology involving the identification of cellular receptors, enzymes, and pathways involved in tumor growth and immortality have resulted in the development of biologically targeted drugs. The use of targeted therapies in conjunction with RT promises to enhance the therapeutic ratio by increasing the efficacy of RT without significantly increasing treatment-related side effects.

As individual tumor mutations and molecular markers are better understood, it may also be possible to improve patient selection by determining which patients will benefit from a given targeted therapy.

\section{Concomitant Chemoradiation}

The MACH-NC, meta-analysis of concomitant chemotherapy along with RT in head and neck cancer published almost a decade ago included 63 randomized trials conducted over a nearly 30 -year period in more than 10,000 patients with a median follow-up of 6 years. The introduction of platinum-based chemotherapy to radiation reduced the relative risk of death to 0.89 . Concomitant approaches yielded the greatest absolute benefit in the 5-year OS rate at $8 \%$ compared with only $1 \%$ for adjuvant approaches and $2 \%$ for induction. The $\mathrm{P}$ value for improved survival with concomitant chemoradiation was $<0.0001$. $^{2}$

An update of MACH-NC meta-analysis published recently included 24 additional trials for a total of nearly 18,000 patients. Median follow-up was 5 years and in this more homogeneous population, an $8 \%$ absolute improvement (4.5\% at 5 years in the updated analysis) in 
Table 1. Data from important chemoradiation trials

\begin{tabular}{lcccccc}
\hline & $N$ & $F /$, years & $R T$ (Control) & CT/RT & P Value & Agents used \\
\hline French trial & 226 & 3 & 31 & 51 & 0.002 & Carbo/5-FU \\
German trial & 270 & 3 & 24 & 48 & $<.0003$ & CDDP/5-FU/LV \\
NP intergroup & 193 & 3 & 47 & 78 & 0.005 & cDDP $\rightarrow$ DDP/5-FU \\
Duke U & 116 & 5 & 28 & 42 & 0.05 & cDDP/5-FU \\
Intergroup & 199 & 3 & 23 & 37 & 0.01 & cDDP \\
Greek & 83 & 3 & 18 & 52 & $<0.001$ & CDDP \\
\hline
\end{tabular}

Note: cDDP—Cisplatin, 5FU—Flurouracil, Carbo Carboplatin, LV—Leucovorin, RT—Radiation therapy, CT/RT—Chemoradiation, DFS—Disease free survival, OS-Overall survival.

Table 2. Results from RTOG 91-11 trial $^{4}$

\begin{tabular}{lccc}
\hline Arm & $c D D P / 5-F U \rightarrow R T$ & $R T / c D D P$ & $R T$ \\
\hline Enrolled, n (evaluable) & $180(173)$ & $182(172)$ & $185(173)$ \\
2-years laryngectomy FS, \% & 59 & 66 & 53 \\
5-years DMFS,\% & 85 & 88 & 78 \\
5-years DFS,\% & 38 & 36 & 27 \\
5-years OS,\% & 55 & 54 & 56 \\
\hline
\end{tabular}

Note: cDDP-Cisplatin, 5FU—Flurouracil, RT-Radiation therapy, DFS-Disease free survival, OS-Overall survival.

long-term survival for combined modality approaches, specifically concurrent chemoradiation was observed. ${ }^{3}$ There were no differences in the survival benefit based on anatomic site, including oral cavity, oropharynx, hypopharynx, or larynx. There were also no major differences regarding $\mathrm{T}$ or $\mathrm{N}$ staging. However, age clearly made a difference: patients younger than 50 years of age had a $24 \%$ absolute improvement in survival, but that survival benefit tended to decrease as patients aged; those older than 70 years of age had only a 3\% improvement in survival.

Several trials in locally advanced head and neck cancers have evaluated radiation alone $v s$ radiation and concomitant platinum-based chemotherapy (Table 1), often including cisplatin either alone or combined with 5-fluorouracil. In each case, an approximately 15 to $30 \%$ absolute improvement in long-term survival is obtained with combined chemoradiation. These are some of the most impressive improvements in survival observed in solid tumor oncology.

Forastiere and colleagues published the results of a major phase III trial examining the role of chemotherapy and radiation for organ preservation in advanced laryngeal cancer. RTOG Study 91-11 compared radiation alone with concurrent radiation and platinum chemotherapy vs what many consider to be the control arm, induction platinum and 5-fluorouracil followed by definitive local radiation. To enroll, patients had to have biopsy-proven, previously untreated stage III or IV squamous cell carcinoma of either the glottic or supraglottic larynx, good performance status

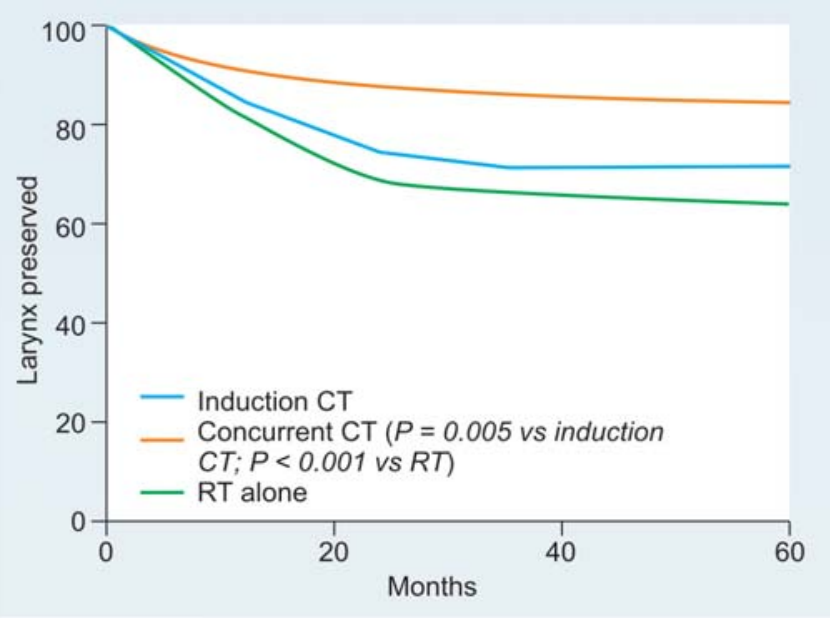

Fig. 2: Advanced laryngeal cancer: preservation ${ }^{4}$

(Karnofsky score $\geq 60$ ), no evidence of distant metastases, and potentially resectable tumors. The primary endpoint was survival with preservation of laryngeal function. In total, 547 patients were accrued and the demographics were fairly standard for a larynx population: the median age was slightly younger than 60 years, the majority had good performance status, $68 \%$ had supraglottic tumors, and $28 \%$ had N2 or N3 involvement. ${ }^{4}$

The 2-year laryngectomy-free survival rate was significantly higher in the concomitant platinum/radiation therapy arm: $66 \%$ vs 59\% for the induction approach and 53\% for radiation alone (Table 2 and Fig. 2). Toxicity was also much greater with concomitant cisplatin and radiation, particularly nausea, vomiting, and esophagitis. The addition 
of platinum therapy to radiation led to a statistically significant improvement in laryngectomy-free survival, with a $P$ value of 0.01 . There was, however no significant difference in OS. ${ }^{4}$

\section{Sequential Chemoradiation}

An alternative approach is to use induction therapy to cytoreduce tumors and then use local modalities after the tumor volume has decreased. The MACH NC analysis identified an $8 \%$ improvement in 5-year survival when chemotherapy was part of the CRT treatment regimen and a 5\% improvement with cisplatin and 5-FU (PF) induction chemotherapy (Table 3). ${ }^{2}$ A follow-up analysis that included an additional 23 trials for a total of over 16,000 patients confirmed a $5 \%$ benefit at 5 years in patients who had received $\mathrm{PF}$ induction chemotherapy as part of their regimen. ${ }^{5}$

Data from randomized trials in patients with stage 3 and 4 locally advanced laryngeal cancer have demonstrated that induction chemotherapy with PF followed by radiation in cases when a response to the chemotherapy regimen can be achieved is equivalent to surgery and resulted in a $64 \%$ rate of organ preservation. ${ }^{6}$

In the past 5-10 years, there have been a number of studies adding taxanes to standard 5-fluorouracil/platinum induction regimens. Most of these studies have featured docetaxel. With one exception, these studies used subsequent radiation alone or radiation with low-dose weekly platinum. In each study, the addition of a taxane was associated with a significant improvement in local control rates, in response rates, both partial and complete responses, and in progression-free survival (PFS). At least two studies also demonstrated a significant improvement in OS and we will review those studies now.

The first study was conducted by Vermorken and colleagues comparing TPF with PF followed by radiation; surgery was used as salvage treatment. The trial enrolled patients with unresectable head and neck cancer. The doses used in the standard arm were $100 \mathrm{mg} / \mathrm{m}^{2}$ of cisplatin on Day 1 and $1 \mathrm{~g} / \mathrm{m}^{2}$ daily continuous infusion of 5 -fluorouracil on Days 1-5 for up to four 21-day cycles. In the experimental arm, docetaxel was added at $75 \mathrm{mg} / \mathrm{m}^{2}$ on Day 1 and both cisplatin and 5-fluorouracil doses were attenuated. Patients had the option of either going on to conventional once-daily fractionated radiation or accelerated or hyperfractionated regimes with a concomitant boost at the conclusion of radiation. An improvement in median PFS was observed with TPF vs PF $(P=0.007)$ and that translated into an OS advantage with a log-rank P value of 0.02 (Fig. 3). ${ }^{7}$

Table 3. Effects of chemotherapy on survival at 5 years from the meta-analysis ${ }^{5}$

\begin{tabular}{lccrr}
\hline Trial category & Number of trials & Number of patients & Difference (\%) & $P$ value \\
\hline All trials & 65 & 10850 & +4 & $<0.0001$ \\
Adjuvant & 8 & 1854 & +1 & 0.74 \\
Induction & 31 & 5269 & +2 & 0.10 \\
Cisplatin and 5-FU & 15 & 2487 & 0 & 0.01 \\
Other chemotherapy & 16 & 2782 & +8 & 0.91 \\
Concomitant & 26 & 3727 & 0.0001 \\
\hline
\end{tabular}
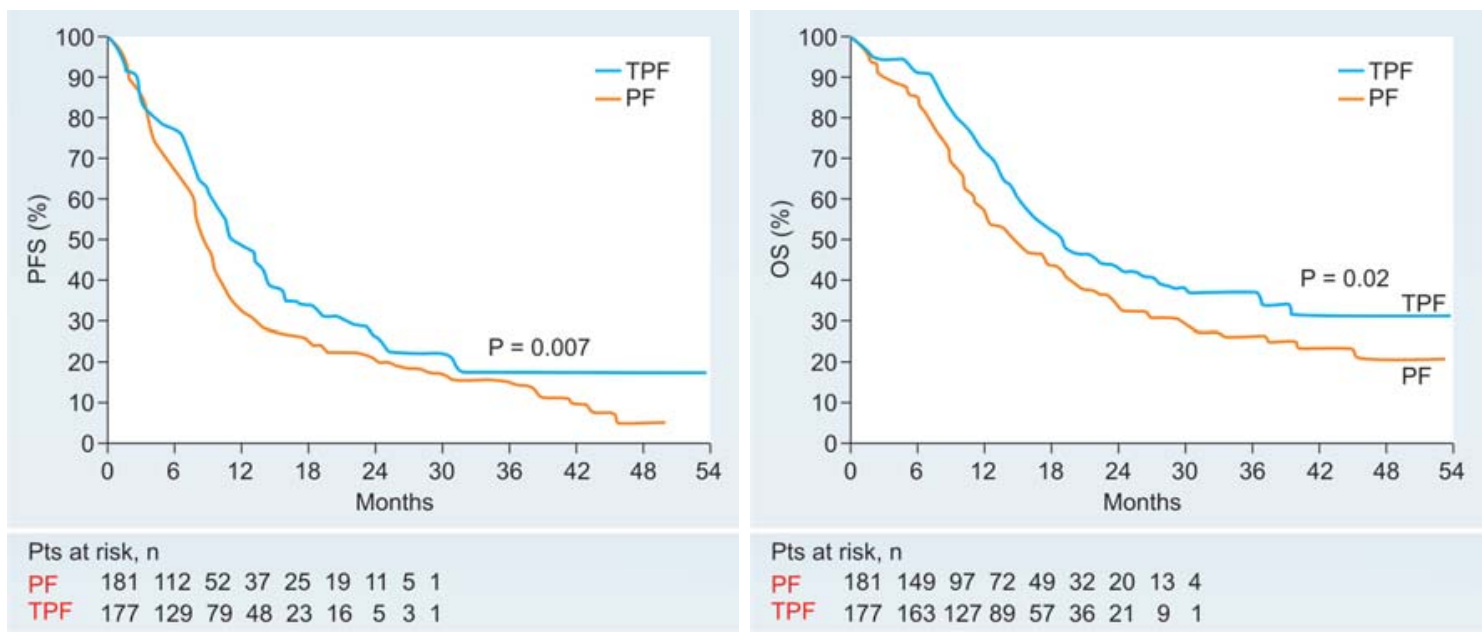

Fig. 3: PFS and OS in TAX 323 study $^{7}$ 


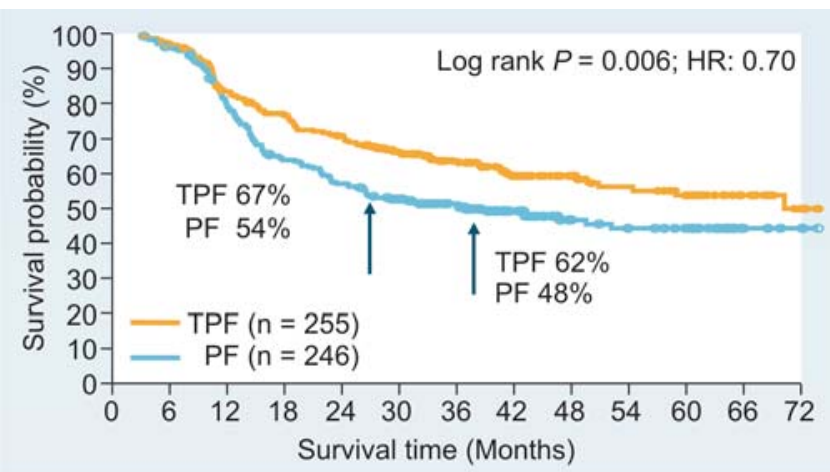

Pts at risk, $\mathrm{n}$

TPF $\quad \begin{array}{lllllllllllll}255 & 234 & 196 & 17 & 163 & 136 & 105 & 72 & 52 & 45 & 37 & 20 & 11\end{array}$

PF $\quad \begin{array}{lllllllllllll}246 & 223 & 169 & 6 & 130 & 107 & 85 & 57 & 36 & 32 & 28 & 10 & 7\end{array}$

Fig. 4: Kaplan Meir survival curves in TAX 324 study $^{8}$

A similar study, TAX 324, was conducted by Posner and colleagues, also comparing TPF with PF. Unlike the study by Vermorken and colleagues, the doses of 5fluorouracil and cisplatin in the experimental arm were not attenuated. A significant improvement in OS with TPF vs PF was also observed in this trial: the 3-year OS rates were $62 \%$ vs $48 \%$, respectively, with a very impressive $P$ value of 0.006 and an HR of 0.70 (Fig. 4). ${ }^{8}$

\section{Cetuximab with Radiation}

The only approved agent in head and neck cancer that targets EGFR is cetuximab. Cetuximab is a chimerized antibody exclusive for EGFR and its heterodimers. It prevents repair and survival of tumor cells that have been damaged by the effects of chemotherapy and radiation, potentiates apoptosis, inhibits cell cycle progression, decreases the production of angiogenic factors, and ultimately inhibits tumor invasion and metastases.

Work by Milas and colleagues has shown synergy between cetuximab and radiation. In A431 xenografts, multiple doses of cetuximab grafted onto a single dose of radiation led to radiation enhancement ratios that were nearly 4-fold higher than with radiation alone. ${ }^{9}$

These observations in the preclinical arena ultimately led to phase I, phase II, and phase III studies evaluating the role of cetuximab in the context of standard radiation in head and neck cancer.

Bonner and colleagues published the results of this critical phase III trial 3 years ago. Patients were randomized to radiation alone or to radiation plus concomitant cetuximab. To be eligible, patients had to have locoregionally advanced squamous cell carcinoma of either the oropharynx, hypopharynx, or larynx, and they needed to be candidates for definitive potentially curative radiotherapy with measurable disease and no previous treatment. The primary endpoint was duration of locoregional control. Secondary endpoints included OS, toxicity, and quality of life. ${ }^{10}$

Patients were well matched in both treatment arms. Median age was approximately 57 years. There was no age cutoff in this trial; patients older than 80 years of age were enrolled. The ratio of men to women was approximately $4: 1$. Oropharynx was the primary tumor site in approximately $60 \%$ of patients whereas the larynx was the primary tumor site in approximately $25 \%$ of patients. Among patients who had their tumors assayed for EGFR expression, expression rates were uniform in both treatment arms; EGFR was detectable in approximately $80 \%$ of patients, and it was unknown in another $20 \%$ with insufficient tumor specimens.

The only safety signal in this trial was skin reaction, as one would expect with an agent that targets EGFR. The incidence of grade 3/4 acneiform rash was almost 2-fold higher in the cetuximab arm (34\% compared with 18\%). There was no obvious exacerbation of radiation-induced mucositis by contrast to studies evaluating platinum therapy in the same setting; nor was there any obvious increase in the incidence of dysphasia, xerostomia, or fatigue. There were, as one would expect, infusion reactions associated with cetuximab that would not be seen in the control arm, but only $3 \%$ of patients experienced grade $3 / 4$ infusion reactions.

The addition of cetuximab was associated with a significant improvement in locoregional control at both 1 and 2 years, with a log-rank $P$ value of 0.02 . No significant improvement in the incidence of distant recurrences or second primary malignancies was observed in the combination arm, although there was a trend favoring the cetuximab arm. The cetuximab arm demonstrated an 8\% advantage in the locoregional control rate at 2 years and a near doubling in median time to locoregional progression from 14.9 months in the control group to 24.4 months in the cetuximab group, with a $\mathrm{P}$ value of 0.005 .

This outcome in turn translated into a 20-month improvement in median OS, from 29 months with radiation alone to 49 months with cetuximab plus radiation $(\mathrm{P}=0.018)$. The 2-year OS rate difference was 7\% and the 3-year rate difference was $13 \%$ in favor of cetuximab. At 5 years, the difference in survival rates was maintained at $9 \%$. 
To define further the relative roles of radiotherapy plus cetuximab and CRT in locally advanced disease, a comparison of the efficacy and tolerability of the two approaches is necessary. To date, no phase III trial has directly compared radiotherapy plus cetuximab with CRT. However, between-study comparison of phase III trials shows that the survival advantage over radiotherapy alone of adding cetuximab to radiotherapy is similar to that achieved with CRT (20 months and up to 18 months, respectively). ${ }^{10}$ Bonner and colleagues sought to put the clinical benefit achieved with cetuximab into context with CRT. They conducted a retrospective analysis of data comparing the outcome of 29 patients who had locally advanced HNSCC and were receiving radiotherapy plus cetuximab with that of 103 patients who were receiving cisplatin-based CRT at a single center. No significant difference was shown between radiotherapy plus cetuximab and CRT in terms of 3-year rates of locoregional control (71\% versus $75 \%$ ), distant metastasis-free survival (92\% versus $87 \%$ ), and disease-specific survival (79\% versus 77\%). Three-year overall survival was significantly higher in the radiotherapy plus cetuximab group compared with the CRT group (76\% versus 61\%, P 50.02).

Bonner and colleagues also conducted an analysis of outcomes at a median follow-up of 5 years according to pretreatment characteristics. All patient subgroups demonstrated an improvement with the addition of cetuximab. This improvement was pronounced in patients with oropharyngeal carcinoma, T1-T3 disease as opposed to $\mathrm{T} 4$, those who received concomitant boost radiation as opposed to once-daily radiation, those with nodal involvement N1-N3, those with better performance status, male patients, those with EGFR expression measuring $\leq 50 \%$, suggesting some potential saturation phenomenon, and finally, patients aged younger than 65 years. ${ }^{11}$
The Radiation Therapy Oncology Group (RTOG) is formally testing whether the addition of cetuximab to standard full-dose platinum and radiation is better than chemoradiation alone. RTOG 0522 randomized patients to concomitant boost radiation with 2 doses of cisplatin per cycle vs full-dose radiation, cisplatin, and cetuximab during the concurrent approach. This trial was originally slated to accrue 720 patients; however, it accrued so rapidly that it was decided based on a pooled survival analysis to increase the accrual to 945 patients. The accrual completed in March 2009 and the results should be available in approximately the next 2 years.

The European investigators are examining targeted therapy with an induction platform that integrates TPF followed by either full-dose platinum and radiation or cetuximab and radiation. This is the only trial that directly compares platinum/radiation therapy with cetuximab/ radiation therapy. Patients with relatively poor responses to induction go on to surgical salvage with total laryngectomy and postoperative radiation. Patients with good responses are randomized to full-dose radiation with either platinum or cetuximab. Preliminary data from this trial, presented by Lefebvre and colleagues at the 2009 Annual Meeting of the American Society of Clinical Oncology, showed far better safety in the cetuximab arm. ${ }^{12}$

\section{Adjuvant Chemoradiation}

There have been 3 relatively recent trials that have compared radiation alone with concurrent platinum and radiation in the adjuvant setting (Table 4). The RTOG study $9501^{13}$ and the European Organization for Research and Treatment of Cancer (EORTC) study 22931 are similar studies; both employed full-dose chemotherapy, in this case cisplatin at $100 \mathrm{mg} / \mathrm{m}^{2}$. In both studies, the investigators observed a significant improvement in locoregional control and disease-

Table 4. Results of adjuvant chemoradiation trials ${ }^{13-15}$

\begin{tabular}{llllll}
\hline Trial & $R T(G)$ & $F / U$, months & LRC, \% & DFS, \% & OS, \% \\
\hline RTOG 9501[13] & $\mathrm{n}=459$ & 46 & 81 vs 70 & 33 vs 25 & 45 vs 38 \\
2 2 LN, ECE, + margins & $(60-66)$ & & $(\mathrm{P}=0.01)$ & $(\mathrm{P}=0.04)$ & $(\mathrm{P}=0.19)$ \\
EORTC 22931[14] & $\mathrm{n}=350$ & 60 & 82 vs 69 & 47 vs 36 & 53 vs 40 \\
N2-3, ECE, + margins & $(66)$ & & $(\mathrm{P}=0.007)$ & $(\mathrm{P}=0.04)$ & $(\mathrm{P}=0.002)$ \\
Bachaud[15] + ECE & $\mathrm{n}=83$ & 60 & 70 vs 55 & 45 vs 23 & 36 vs 13 \\
& $(>60)$ & & $(\mathrm{P}=0.05)$ & $(\mathrm{P}<0.02)$ & $(\mathrm{P}<0.01)$ \\
\hline
\end{tabular}

Note: RT—Radiation therapy, F/U—Follow-up, LRC—Locoregional control, DFS—Disease free survival, OS—Overall survival. 
free survival with the addition of platinum-based therapy. In the EORTC trial, a significant improvement in OS was noted. ${ }^{14}$ A provocative trend towards improved OS was also evident in the RTOG study. A much smaller study by Bachaud and colleagues compared weekly platinum plus radiation with radiation alone and they also observed a significant improvement in locoregional control, diseasefree survival, and OS. ${ }^{15}$

\section{CONCLUSION}

Increasing numbers of clinical studies are identifying molecular markers that indicate patient prognosis and the likelihood of a tumor responding to particular treatments or that provide an opportunity for direct, potentially therapeutic targeting. The use of biologic agents directed at potentially therapeutic molecular targets now forms an integral part of treatment of several malignancies. The first, and currently only, biologic agent to be given regulatory approval in Europe or the United States for the treatment of locally advanced HNSCC in combination with radiotherapy is the EGFR antagonist, cetuximab. The combination of cetuximab and radiotherapy led to statistically and clinically significant benefits over radiotherapy alone and was well tolerated. It is up to the treating physician to decide which is the most appropriate approach. Because the two treatment approaches are not being compared in a definitive trial, we must turn to retrospective comparisons for interpretation, and such comparisons indicate that the efficacy of radiotherapy plus cetuximab, and CRT, are similar. Radiotherapy plus cetuximab has in its favor a better tolerability profile than CRT. The efficacy results and safety profile observed so far strongly suggest that adding cetuximab to radiotherapy significantly increases the therapeutic index of this latter modality. The cost of treatment will also be a consideration.

Results from ongoing trials will tell us if the addition of cetuximab improves the efficacy of CRT without exacerbating toxicities.

\section{REFERENCES}

1. Mierzwa, Nyati, Morgan, et al. Recent Advances in Combined Modality Therapy. The Oncologist 2010;15:372-81.

2. Pignon JP, Bourhis J, Domenge C, et al. Chemotherapy added to locoregional treatment for head and neck squamous cell carcinoma: three meta-analyses of updated individual data. MACH-NC collaborative group. Meta-analysis of chemotherapy on head and neck cancer. Lancet 2000;355:949-55.

3. Pignon JP, Bourhis J, Domenge C, et al. Radiother Oncol 2009;92:4-14.

4. Forastiere AA, et al. N Engl J Med 2003;349:2091-98.

5. Monnerat C, Faivre S, Temam S, et al. End points for new agents in induction chemotherapy for locally advanced head and neck cancers. Ann Oncol 2002;13:995-1006.

6. The Department of Veterans Affairs Laryngeal Cancer Study Group. Induction chemotherapy plus radiation compared with surgery plus radiation in patients with advanced laryngeal cancer. N Engl J Med 1991;324:1685-90.

7. Vermorken JB, et al. N Engl J Med 2007;357:1695-1704.

8. Posner MR, et al. N Engl J Med 2007;357:1705-15.

9. Milas L, et al. Int J Radiat Oncol Biol Phys 2004;58:966-71.

10. Bonner JA, et al. N Engl J Med 2006;354:567-78.

11. James A Bonner, Paul M Harari, Jordi Giralt. Radiotherapy plus cetuximab for locoregionally advanced head and neck cancer: 5-year survival data from a phase 3 randomised trial, and relation between cetuximab-induced rash and survival. Lancet Oncol 2010;11:21-28.

12. Lefebvre J, et al. ASCO 2009. Abstract 6010.

13. Cooper JS, et al. N Engl J Med 2004;350:1937-44.

14. Bernier J, et al. N Engl J Med 2004;350:1945-52.

15. Bachaud JM, et al. Int J Radiat Oncol Biol Phys 1991;20: 243-46. 